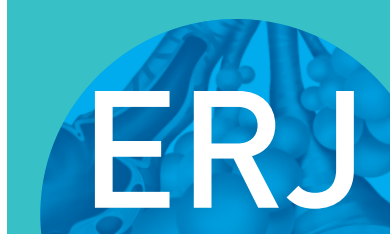

open research

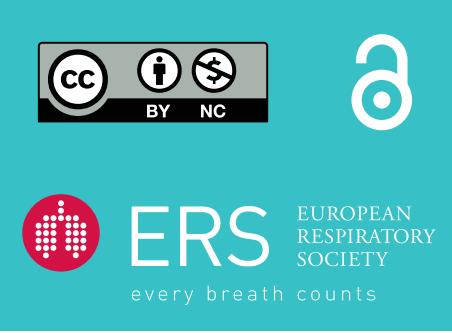

\section{Focal bronchial dilatations after thermoplasty for severe asthma}

\section{To the Editor:}

Bronchial thermoplasty (BT) is a non-pharmacological alternative treatment for severe asthma $[1,2]$. BT consists of applying radiofrequency energy to the airways that are distal to the lobar bronchi and $>3 \mathrm{~mm}$, to reduce airway smooth muscle mass and bronchial hyperreactivity. However, concerns have been raised about long-term safety, especially risk of bronchial stenosis or bronchiectasis. Indeed, morphological changes in bronchial tubes, such as bronchiectasis or widening of the airways, have been occasionally described during follow-up [3-7]. However, bronchial changes have not been systematically assessed by computed tomography $(\mathrm{CT})$, especially in large prospective cohorts $[2,3,8,9]$.

In this retrospective observational study, we included patients with severe asthma who underwent BT (three procedures, every 3 weeks) in our centre between January 2013 and December 2017 and who had at least one chest CT scan before BT and, at the earliest, 4 months after the last procedure. Pre-BT CT scan, the first post-BT CT scan taken at least 4 months after the procedure and the last available CT scan were visually analysed and compared by three operators, including a specialised radiologist, who were blinded to the clinical response. Analysis focused on the first appearance of new bronchial dilatations after BT, and their location, shape and evolution on successive CT scans.

We identified 26 asthmatic patients who fulfilled the inclusion criteria. Clinical characteristics are summarised in figure 1a. Mean \pm SD age was $47 \pm 11$ years. 15 (58\%) patients were female and $12(46 \%)$ were ex- or current smokers. Mean \pm SD $\mathrm{FEV}_{1}$ before BT was $65 \% \pm 17 \%$. Mean \pm SD follow-up after BT was $30.1 \pm 19.6$ months (range 6-72 months).

Before BT, four (15\%) patients had mild diffuse bronchiectasis, four (15\%) had cylindrical bronchiectasis only located in the middle lobe, and diffuse bronchial thickening was observed in $16(61 \%)$. After BT, one or more new focal bronchial dilatations was detected on CT in $15(58 \%)$ patients, with a mean \pm SD number of $4.0 \pm 2.7$ dilatations per patient. Most dilatations were tubular, with an unusual fusiform shape, and $1-2 \mathrm{~cm}$ in length (figure $1 \mathrm{~b}$ ), a few were shorter with a round shape (figure 1c). Bronchial dilatations were detected on the first post-BT CT scan (performed at a mean \pm SD of $16 \pm 9$ months post-BT (range 4-41 months)) in 13 out of 15 patients and on the second CT scan in the remaining two patients. 17 patients (65\%) had more than one CT scan after BT during their follow-up; nine had new focal bronchial dilatations diagnosed. In this sub-group, neither the number nor the size (diameter and length) of focal abnormalities changed over a mean \pm SD follow-up of $41 \pm 21$ months (range 15-69 months). For patients without new focal bronchial dilatations, the mean CT scan follow-up was $41 \pm 23$ months (range 11-72 months).

Bronchial dilatations were located in fourth- to fifth-order bronchi, in the left lower lobe in $40 \%$ of cases, right upper lobe in $30 \%$ and right lower lobe in $25 \%$. The mean diameter was $4.5 \pm 0.9 \mathrm{~mm}$. The 15 patients with new dilatations after BT did not differ from their counterparts in terms of baseline characteristics (figure 1a). The oral cortico-sparing effect, reduction in exacerbations and hospitalisations and increase in Asthma Control Test scores at 12 months after BT were similar between the two groups (figure 1a). The number of activations during the procedure was equivalent. We did not find any correlation between the number of activations in a lobe and number of focal bronchial dilatations.

@ERSpublications

Focal bronchial dilatations develop after bronchial thermoplasty (BT) in $58 \%$ of patients with severe asthma. This suggests a need for systematic evaluation by CT scan after BT, with specific focus on bronchial dilatation development. https://bit.ly/2AYuhMj

Cite this article as: Thibaut de Ménonville C, Debray M-P, Alavoine L, et al. Focal bronchial dilatations after thermoplasty for severe asthma. ERJ Open Res 2020; 6: 00117-2020 [https://doi.org/ $10.1183 / 23120541.00117-2020]$.

Copyright $\odot$ ERS 2020. This article is open access and distributed under the terms of the Creative Commons Attribution NonCommercial Licence 4.0. 
a)

Parameter

Subjects $\mathbf{n}$

Females $n(\%)$

Age years

Body mass index $\mathrm{kg} \cdot \mathrm{m}^{-2}$

Baseline prebronchodilator $\mathrm{FEV}_{1} \mathrm{~L}$

Baseline prebronchodilator $\mathrm{FEV}_{1} \%$

Post-BT prebronchodilator $\mathrm{FEV}_{1} \mathrm{~L}$

\section{Treatments}

Baseline dose of ICS $\mu \mathrm{g} \cdot$ day $^{-1 \#}$

Post-BT dose of ICS $\mu \mathrm{g} \cdot \mathrm{day}^{-1 \#}$

On maintenance OCS before BT $n(\%)$

OCS withdrawal after BT $n[\%)$

Asthma control

Baseline ACT score

Post-BT ACT score

ACT change after BT

Baseline annual rate of exacerbations

Post-BT annual rate of exacerbations

Baseline annual rate of hospitalisation for asthm

Post-BT annual rate of hospitalisation for asthma

\section{Post-BT complication}

Haemoptysis $n(\%)$

Respiratory infection $n[\%]$

\section{Follow-u}

CT-scans performed after BT n

Delay between BT and first CT scan

Delay between BT and last CT scan

26
$15(58)$

$46.9 \pm 11.2$

$29.7 \pm 7.5$

$2.0 \pm 0.7$

$65.2 \pm 17$

$1.9 \pm 0.6$

$2500 \pm 866$

$1935 \pm 834$

17 (65)

4 (15)

$9.0 \pm 3.4$

$15.1 \pm 6.7$

$+6.0+7.2$

$7.4 \pm 3.8$

$4.6 \pm 5.0$

$2.0 \pm 2.8$

$0.15+0.5$

$$
\begin{array}{r}
2(8) \\
6(23)
\end{array}
$$

$1.88 \pm 0.76$

$13.1 \pm 5.5$

$30.1 \pm 19.6$
Focal bronchiectasis 15

$$
\begin{gathered}
15 \\
10(67)
\end{gathered}
$$

$10(67)$
$48.1 \pm 2.6$

$28.3 \pm 1$.

$2.0 \pm 0.2$

$67 \pm 5$

$1.9 \pm 0.2$

\section{$2831 \pm 411$}

$1910 \pm 284$

$9(60)$

2 (22)

$10.9 \pm 1.1$

$15.7 \pm 2.0$

$+4.8 \pm 2.4$

$7.8 \pm 0.9$

$3.7 \pm 1.6$

$2.2 \pm 1.0$

0

$1(6)$

$4(26)$

$1.7 \pm 0.2$

$12.6 \pm 1.6$

$22.1 \pm 3.1$ b)

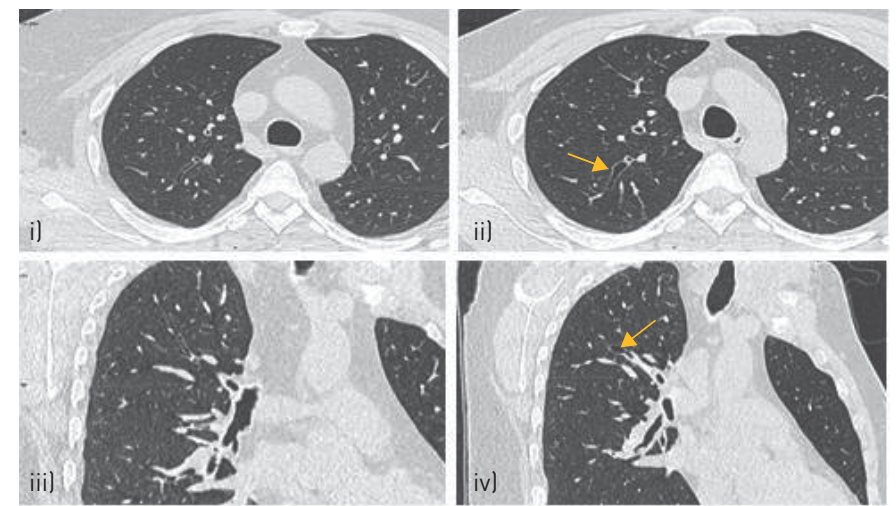

$\begin{array}{ll}7.2 \pm 0.7 & 0.01\end{array}$

$14.5 \pm 2.3 \quad 0.70$

$+7.3 \pm 2.1 \quad 0.45$

$\begin{array}{ll}8.1 \pm 1.4 & 0.88\end{array}$

$5.2 \pm 1.5 \quad 0.52$

$1.8 \pm 0.6$

$0.3 \pm 0.2$

1 (9)

2 (18)

$2.1 \pm 0.2$

$13.8 \pm 1.4$

$41 \pm 23.1$
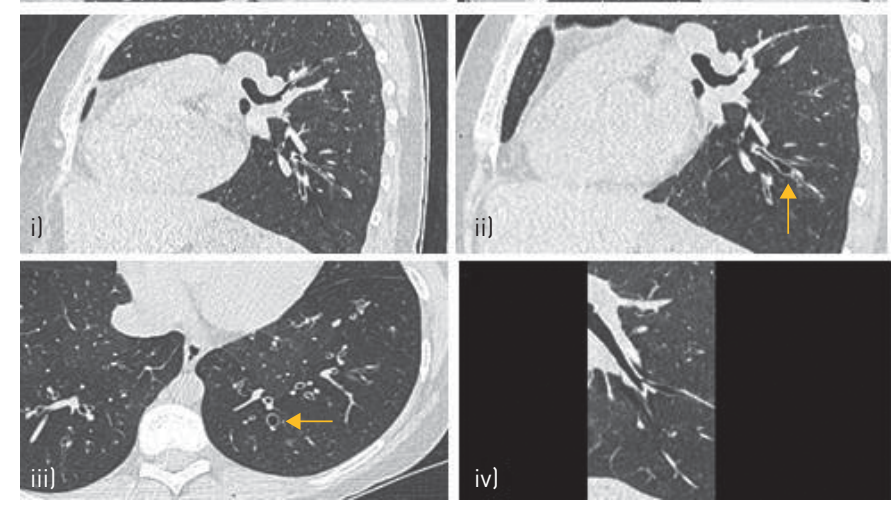

Patient characteristics. Comparison of baseline and 12-month post-bronchial thermoplasty (BT) parameters between patients with or without the appearance of new bronchial dilatations (bronchiectasis). Data are presented as mean \pm SD, unless otherwise stated. FEV ${ }_{1}$ : forced expiratory volume in $1 \mathrm{~s}$; ICS: inhaled corticosteroids; OCS: oral corticosteroids; ACT: asthma control test; CT: computed tomography. " : beclomethasone equivalents; ${ }^{1}$ : during the first year after BT. b) il and iii) Axial and coronal planes of CT before BT. ii) and iv) Axial and coronal planes of CT of the same patient 2 years after BT, showing a fusiform bronchial dilatation (arrows). c) i) Coronal plane of CT before BT. ii) and iii) Coronal and axial planes of CT of the same patient 3 years after BT, showing a sacciform bronchial dilatation (arrow). iv) CT reconstruction showing the focal bronchial dilatation. 
Bronchial changes after BT have been poorly explored. In the AIR2 trial [5], only 93 of the 190 patients had a CT scan after the procedure. Bronchial dilatations were observed in 3\% [5]. Other smaller studies reported bronchial dilatations in $8 \%$ [7] and 15\% [4] of patients, which is much lower than that observed in the present study (58\%). The focal bronchial dilatations such as we describe, some being subtle, may have been missed without careful examination in previous studies.

Mild diffuse bronchiectasis is observed in $25 \%$ to $67.5 \%$ of patients with severe asthma [10]. These cases differ from the focal fusiform dilatations we observed, whose aspect has never been described in asthma. These changes can be detected as soon as 4 months after the last procedure and were never observed in the untreated middle lobe and always occurred in fourth- to fifth-order bronchi. No bronchial stenosis was observed, although it was not quantitatively assessed [6]. These observations suggest a limited, local, thermal energy-induced tissular damage, such as that discussed with the development of early peribronchial consolidations [4], rather than a fibrotic wound healing. BT-induced structural changes, such as focal destruction of airway smooth muscle, replaced by loose connective tissue, or bronchial nerve ending damage, as previously described [9], may contribute to reduced bronchial tone and global increase in airway diameter.

Focal dilatations occurred in more than half of our patients after BT but did not modify the clinical response, which is comparable to previously reported series. They were not associated with an increased rate of side-effects, such as infections or haemoptysis, over a 12-month clinical follow-up, or with poorer lung function. Two cases (7\%) of haemoptysis were reported after BT, one in each group (new dilatations and no new dilatations) occurring in the early post-procedure phase, and were not related to bronchial dilatations.

This study has some limitations due to its retrospective design, the relatively small number of patients and different follow-up among patients, which does allow for proper evaluation of BT safety. We are confident that these focal dilatations are related to the procedure and not to the natural history of airway changes in severe asthma. Indeed, some other investigators in France have also observed such changes at the same delay after the procedure (N. Guibert and L. Guilleminault, CHU de Toulouse, and C. Fournier, CHRU de Lille; personal communication). A control group of patients without BT would have not been matched in terms of airway inflammatory pattern, a strong determinant for airway remodelling, because BT was mostly performed in our centre in patients not eligible for biological agents during the study period. Given the observations reported here, this indication is still current in our hospital, in accordance with recommendations from French health authorities. Special attention is given to diffuse bronchiectasis, which when diffuse and even mild contraindicates the procedure.

Although we did not find any correlation between airway changes and number of activations, our findings argue for BT protocol optimisation, as was already suggested to improve treatment efficiency [11]. Whether limiting the number of treated lobes may reduce the incidence of bronchial dilatations while maintaining equivalent efficacy, although hypothetical, could be investigated. Indeed, although we did not identify clinical complications related to bronchial dilatations, we cannot exclude the possibility of longer-term complications or a significant rate of complications in a larger effective sample. We suggest that systematic evaluation by CT scan should be performed after BT, with specific focus on the development of bronchial dilatations.

Charlotte Thibaut de Ménonville ${ }^{1,6}$, Marie-Pierre Debray ${ }^{2,6}$, Loubna Alavoine ${ }^{3}$, Marie-Christine Dombret ${ }^{4}$, Antoine Khalil ${ }^{2}$, Pierre-Yves Brillet ${ }^{5}$, Michel Aubier ${ }^{1}$ and Camille Taillé ${ }^{1}$

${ }^{1}$ Groupe Hospitalier Universitaire AP-HP Nord-Université de Paris, Hôpital Bichat, Service de Pneumologie et Centre de Référence constitutif des Maladies Pulmonaires Rares, Inserm UMR 1152, Paris, France. ${ }^{2}$ Groupe Hospitalier Universitaire AP-HP Nord-Université de Paris, Hôpital Bichat, Service de Radiologie, Paris, France. ${ }^{3}$ Groupe Hospitalier Universitaire AP-HP Nord-Université de Paris, Hôpital Bichat, Centre d'Investigation Clinique, Paris, France. ${ }^{4}$ Groupe Hospitalier Universitaire AP-HP NordUniversité de Paris, Hôpital Bichat, Service de Pneumologie et Centre de Référence constitutif des Maladies Pulmonaires Rares, Paris, France. ${ }^{5}$ Université Paris 13, INSERM UMR 1272 "Hypoxie et Poumon", Assistance Publique-Hôpitaux de Paris, Hôpital Avicenne, Service de Radiologie, Bobigny, France. ${ }^{6}$ These authors contributed equally.

Correspondence: Camille Taillé, Service de Pneumologie, Hôpital Bichat 46 rue Henri Huchard, 75018 Paris, France. E-mail: camille.taille@aphp.fr

Received: 4 March 2020 | Accepted after revision: 7 July 2020 
Acknowledgements: We thank Clément Fournier (CHRU de Lille, Lille, France), Laurent Guilleminault and Nicolas Guibert (CHU de Toulouse, Toulouse, France) for helpful discussions and sharing experience, and Laura Smales (BioMedEditing, Toronto, Canada) for help with English editing.

Conflict of interest: C. Thibaut de Ménonville has nothing to disclose. M-P. Debray reports personal fees from PneumRx (Boston scientific), personal fees and nonfinancial support from Boehringer Ingelheim, and nonfinancial support from Roche, outside the submitted work. L. Alavoine has nothing to disclose. M-C. Dombret has nothing to disclose. A. Khalil has nothing to disclose. P-Y. Brillet has nothing to disclose. M. Aubier reports grants from GSK, AstraZeneca and Boston Scientific, and grants and personal fees from Chiesi, outside the submitted work. C. Taillé reports personal fees from and that she was a study investigator for AstraZeneca; personal fees from and that she was a study investigator for Boehringer; personal fees from Chiesi; grants and personal fees from, and that she was a study investigator for GSK; personal fees from and that she was a study investigator for Novartis; personal fees from Teva; and that she was a study investigator for Sanofi, all outside the submitted work.

\section{References}

$1 \quad$ Kheir F, Majid A. Bronchial thermoplasty: a nonpharmacologic therapy for severe asthma. Clin Chest Med 2018; 39: 261-269.

2 Castro M, Rubin AS, Laviolette M, et al. Effectiveness and safety of bronchial thermoplasty in the treatment of severe asthma: a multicenter, randomized, double-blind, sham-controlled clinical trial. Am J Respir Crit Care Med 2010; 181: 116-124.

3 Qiu M, Lai Z, Wei S, et al. Bronchiectasis after bronchial thermoplasty. J Thorac Dis 2018; 10: E721-E726.

4 Debray M-P, Dombret M-C, Pretolani M, et al. Early computed tomography modifications following bronchial thermoplasty in patients with severe asthma. Eur Respir J 2017; 49: 1601565.

5 Wechsler ME, Laviolette M, Rubin AS, et al. Bronchial thermoplasty: long-term safety and effectiveness in patients with severe persistent asthma. J Allergy Clin Immunol 2013; 132: 1295-1302.

6 Grenier PA, Fetita CI, Brillet P-Y. Quantitative computed tomography imaging of airway remodeling in severe asthma. Quant Imaging Med Surg 2016; 6: 76-83.

7 d'Hooghe JNS, van den Berk IAH, Annema JT, et al. Acute radiological abnormalities after bronchial thermoplasty: a prospective cohort trial. Respir Int Rev Thorac Dis 2017; 94: 258-262.

8 Langton D, Sloan G, Banks C, et al. Bronchial thermoplasty increases airway volume measured by functional respiratory imaging. Respir Res 2019; 20: 157.

9 Pretolani M, Bergqvist A, Thabut G, et al. Effectiveness of bronchial thermoplasty in patients with severe refractory asthma: clinical and histopathologic correlations. J Allergy Clin Immunol 2017; 139: 1176-1185.

10 Perez-Miranda J, Traversi L, Polverino E. Bronchiectasis in severe asthma: a distinct phenotype? Curr Opin Pulm Med 2019; 25: 71-78.

11 Chernyavsky IL, Russell RJ, Saunders RM, et al. In vitro, in silico and in vivo study challenges the impact of bronchial thermoplasty on acute airway smooth muscle mass loss. Eur Respir J 2018; 51: 1701680. 